\title{
Complete $\mathrm{e}-\mathrm{Zn}$ and $\mathrm{e}-\mathrm{Cd}$ Scattering Experiments
}

\author{
M. PIWIŃSKI* AND Ł. KŁOsOWsKI \\ Institute of Physics, Faculty of Physics, Astronomy and Informatics, \\ Nicolaus Copernicus University in Toruń, Grudziądzka 5, 87-100 Toruń, Poland \\ Doi: 10.12693/APhysPolA.139.236 \\ *e-mail: mariusz.piwinski@fizyka.umk.pl
}

\begin{abstract}
The electron impact coherence parameters for electron impact excitation of $5^{1} P_{1}$ cadmium and $4^{1} P_{1}$ zinc state are presented. The collision process was investigated for the electron energy range from $40 \mathrm{eV}$ to $100 \mathrm{eV}$. The obtained experimental results are presented together with the existing convergent close coupling and relativistic distorted-wave approximation theoretical predictions. The present work is a brief review of our experimental results obtained for the 12th group atoms of the periodic table.
\end{abstract}

topics: zinc, cadmium, electron impact excitation, EICP

\section{Introduction}

The experimental data on collision processes play a significant role in studying planetary atmospheres, stars and interstellar clouds, where the electron collisions with atoms are one of the main mechanisms of energy transfer. For example, the presence of cadmium in interstellar matter provides information about the processes of catching neutrons in nearby stars [1] and quantitative studies on the presence of zinc deliver valuable information on the chemical evolution of the stars [2]. Although there are various experiments involving studies of the collisional processes, in most cases the obtained results are averaged over unobserved parameters. Such research may include measurements of differential cross-sections [3], optical excitation [4-7] and spin asymmetry functions [8, 9]. In 1969, Bederson proposed "a complete scattering experiment" that enables to determine all the scattering amplitudes and obtain a full information (in the quantummechanical sense) about the collision process [10]. Such "complete data" would allow the proposed theoretical models to be tested.

One of the possible implementations of such an idea are electron-photon coincidence measurements in both polarisation [11] and angular correlation versions [12]. Technological progress in research equipment has enabled to use such technique to conduct research on calcium [13], magnesium [14] and strontium [15]. All these elements have in the ground state two valence electrons outside completely closed shells which makes research on them a natural extension of studies on helium [16]. However, a much more interesting subject of research seem to be the atoms from the 12th group, such as zinc and cadmium. Their electron configurations are similar to alkaline earth metals (two valence electrons outside the completely closed shells). Thus, such studies give the possibility for observation of the increasing impact of the fine structure and the spin dependent interactions on the collisional excitation process. This influence could be manifested, for example, by the lack of full coherence of the collision, which can be verified in this type of experiment. Moreover, a comparison of the data for zinc and calcium as well as cadmium and strontium would allow to examine the effect of a closed subshell $(n-1) d^{10}$ on the collision process.

\section{Electron impact coherence parameters}

Considering the collisional excitation of an atom from $S_{0}$ to $P_{1}$ state, the excited one can be described as a coherent superposition of three magnetic sub-states. In a natural coordinate system for which the quantization axis is defined in a direction perpendicular to the collision plane (defined by the momentum vectors of the incident and scattered electron), due to the reflective symmetry, only two sub-states make a non-zero contribution to this superposition. Therefore, the excited $P$ state can be expressed as

$$
|P\rangle=a_{-1}\left|m_{j}=-1\right\rangle+a_{+1}\left|m_{j}=+1\right\rangle,
$$

where $a_{-1}$ and $a_{+1}$ mean the complex coefficients representing the relevant excitation amplitudes.

In such a case, the angular part of the electron charge cloud of the excited atom to $P$ state can be described with an expression

$|\Psi(\vartheta, \varphi)|^{2}=\frac{3}{8 \pi} \sin ^{2}(\vartheta)\left(1+P_{L} \cos (2 \varphi-2 \gamma)\right)$, 


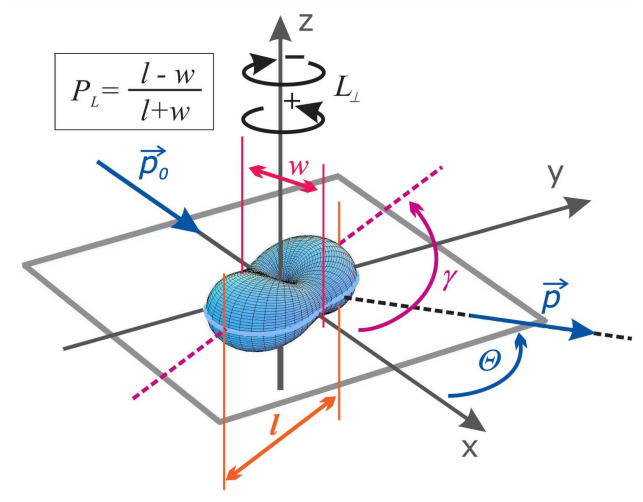

Fig. 1. Example of angular distribution of the electron charge cloud of an atomic $P$ state. The $\boldsymbol{p}_{0}$ and $\boldsymbol{p}$ symbols represent momenta of incoming and scattered electron, $\Theta$ is the scattering angle, $\gamma$ represents the alignment angle and $L_{\perp}$ is the angular momentum transfer parameter. The shape parameter $P_{L}$ can be related to the length $l$ and width $w$ of the cloud.

where $P_{L}$ and $\gamma$ are the shape and the alignment angle parameters [17, 18]. They are defined as

$$
\begin{aligned}
& P_{L}=2\left|a_{-1}^{*} a_{+1}\right|, \\
& \gamma=\frac{1}{2} \arg \left(-a_{-1}^{*} a_{+1}\right) .
\end{aligned}
$$

A complete description of the collision process also needs information about an angular momentum transfer which is described by $L_{\perp}$ parameter

$$
L_{\perp}=\left|a_{+1}\right|^{2}-\left|a_{-1}\right|^{2} \text {. }
$$

The graphic interpretation of these parameters is presented in Fig. 1. Moreover, the coherence of the investigated process can be verified using $P^{+}$parameter defined as:

$$
P^{+}=\sqrt{L_{\perp}^{2}+P_{L}^{2}}
$$

which in a fully coherent case should be equal to one.

\section{Experimental setup}

The full description of the apparatus used for cadmium and zinc studies can be found in our previous papers [19-21]. Briefly, experiment involves the analysis of polarisation of fluorescence from the excited state detected in coincidence with scattered electrons. The atoms are excited from the ground to the investigated state as a result of a collision with electrons with strictly defined energy. Electrons scattered at a given angle $\Theta$ are analysed energetically in such a way that only the ones that excited the atom to the observed state reach the detector. At the same time, in the direction perpendicular to the collision plane, the polarisation of the fluorescence emitted by the atom during the transition from the investigated to the ground state is analysed (see Fig. 2).

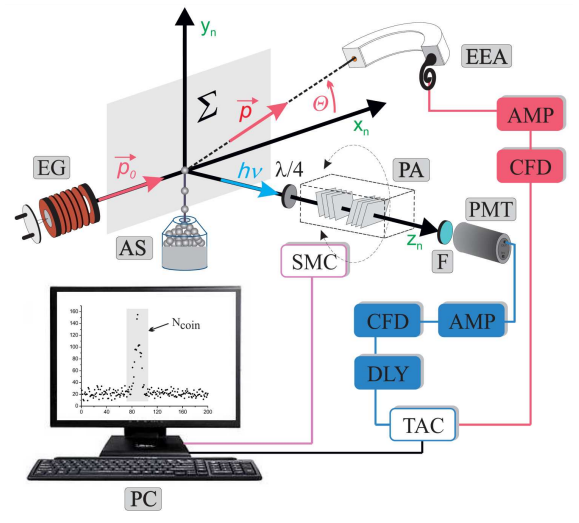

Fig. 2. Schematic outline of the apparatus and geometry of the electron-photon coincidence experiment in the coherence analysis version: EG - electron gun, EEA - electron energy analyser with channel electron multiplier, AS - the source of the atomic beam (Zn or $\mathrm{Cd}$ ), $\lambda / 4$ - zero-order quartz retardation plate, PA - "pile-of-plates" polarisation analyser, F - broadband filter (Zn $214 \mathrm{~nm}$, Cd $229 \mathrm{~nm}$ ), PMT — photomultiplier tube, CFD - constant fraction discriminator, SMC - stepper motor controller, TAC - time-to-amplitude converter, AMP - preamplifier, DLY — delay line, PC - personal computer with multichannel analyser. The scattering plane $\Sigma$ is defined by $\boldsymbol{p}_{0}$ and $\boldsymbol{p}$ which are the momentum vectors of electron before and after the collision, $\Theta-$ the scattering angle.

The time correlation between electron (start) and photon (stop) signals is measured. As a result, the coincidence spectra are determined for various positions of the polarisation analyser axis. Finally, it allows us to determine the number of true coincidences for each of the analyser positions, which leads to the calculation of the Stokes parameter values. Using the formulae below, they can be easily converted into EICP parameters

$$
\begin{aligned}
& P_{\mathrm{L}}=\sqrt{P_{1}^{2}+P_{2}^{2}}, \\
& \gamma=\frac{1}{2} \arg \left(P_{1}+\mathrm{i} P_{2}\right), \\
& L_{\perp}=-P_{3} .
\end{aligned}
$$

Thus, according to expression (6), the coherence parameter $P^{+}$can be also determined as

$$
P^{+}=\sqrt{P_{1}^{2}+P_{2}^{2}+P_{3}^{2}} \text {. }
$$

\section{EICP cadmium results}

The experimentally determined EICPs for a collisionally excited cadmium atom to the $5^{1} P_{1}$ state are presented for electron energy 100, 80 and $60 \mathrm{eV}$. The data are shown graphically together with convergent close coupling (CCC) and relativistic distorted-wave approximation (RDWA) theoretical 
results (see Figs. 3-5). The analysis of all the EICP data sets allows some broader conclusions on general trends and differences between theoretical and experimental results to be drawn:

- In the case of the alignment angle parameter $\gamma$, the CCC data for $100 \mathrm{eV}$ and $80 \mathrm{eV}$ are significantly different from the RDWA predictions, while the results for $60 \mathrm{eV}$ show a much better similarity to the CCC data.

- The agreement between the theoretical and experimental data remains at a similar level for different collision energies.

- The characteristic structures of parameters $P_{L}, \gamma$ and $L_{\perp}$ move towards higher scattering angles with decreasing impact energy. This phenomenon can be intuitively explained as a manifestation of a longer interaction time of the electron with the atom during an impact. This conclusion fully agrees with the first Born approximation (FBA) theory analysed in our previous work [22]. Thus, this effect should also be visible in the case of other elements.

- In the case of the CCC model, the characteristic structures of the $\gamma$ parameter are visible for slightly smaller values of the scattering angle than for the RDWA predictions $\left(\approx 1^{\circ}\right)$.

- In the structure of the shape parameter $P_{L}$ in the range of the scattering angle between $15^{\circ}$ and $40^{\circ}$, all data show only one minimum. Similarly, in the case of the angular momentum transfer parameter $L_{\perp}$ one maximum is visible.

- In the case of all impact energies, there was no significant deviation of the $P^{+}$parameter value from unity which indicates that the examined collision process is coherent [22].

- Both the CCC and RDWA models reproduce all the structures visible in the experimental data, although in the case of RDWA they show much smaller amplitudes.
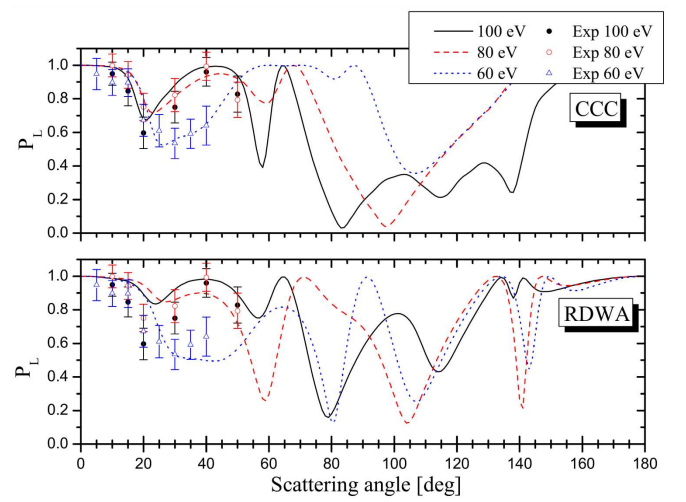

Fig. 3. Shape parameter $P_{L}$ for electronic excitation of $5^{1} P_{1} \mathrm{Cd}$ state. Experimental data: $(\bullet)$ $100 \mathrm{eV},(\circ) 80 \mathrm{eV},(\triangle) 60 \mathrm{eV}$. Theoretical data: (CCC and RDWA): (-) $100 \mathrm{eV},(--) 80 \mathrm{eV},(\cdots)$ $60 \mathrm{eV}[22,25]$.

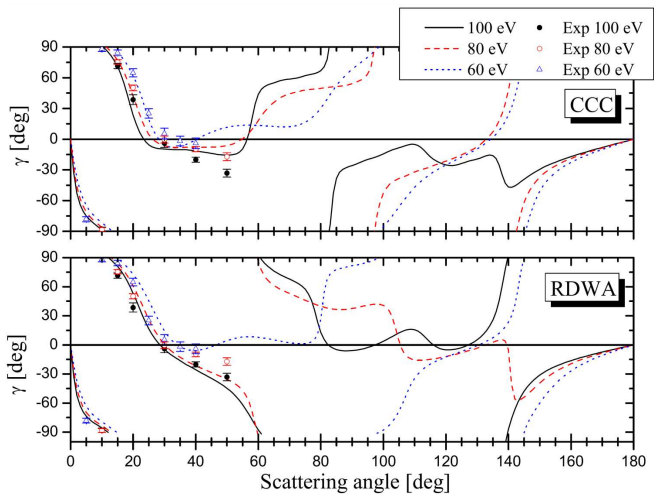

Fig. 4. Alignment angle $\gamma$ for electronic excitation of $5^{1} P_{1} \mathrm{Cd}$ state. Experimental data: $(\bullet)$ $100 \mathrm{eV},(\circ) 80 \mathrm{eV},(\triangle) 60 \mathrm{eV}$. Theoretical data: (CCC and RDWA): (-) $100 \mathrm{eV},(--) 80 \mathrm{eV},(\cdot \cdots)$ $60 \mathrm{eV}[22,25]$.

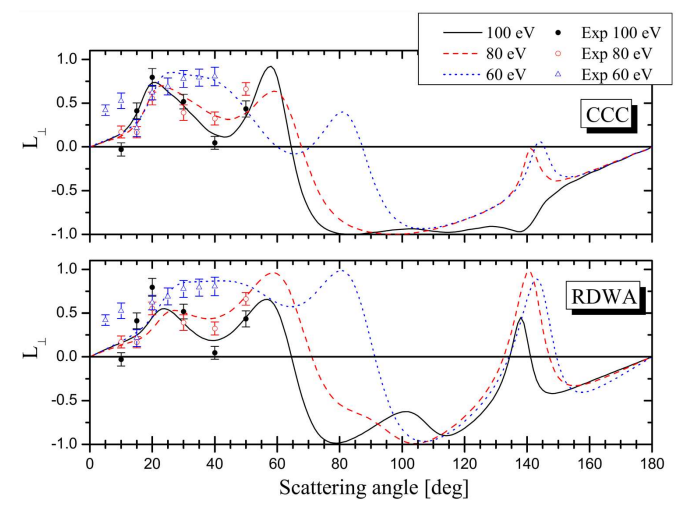

Fig. 5. Angular momentum transfer $L_{\perp}$ for electronic excitation of $5^{1} P_{1} \mathrm{Cd}$ state. Experimental data: $(\bullet) 100 \mathrm{eV},(\circ) 80 \mathrm{eV},(\triangle) 60 \mathrm{eV}$. Theoretical data: (CCC and RDWA): (-) $100 \mathrm{eV},(--) 80 \mathrm{eV}$, $($. . ) $60 \mathrm{eV}[22,25]$.

\section{EICP zinc results}

Figures 6-8 present results of EICPs for a collisionally excited zinc atom to the $4^{1} P_{1}$ state for energy 100, 80, 60 and $40 \mathrm{eV}$ together with $\mathrm{CCC}$ and RDWA theoretical predictions. Similar to the cadmium case, some conclusions could also be drawn:

- In contrast to the cadmium atoms, discrepancies between theoretical and experimental data become greater as the collision energy decreases.

- According to conclusions for cadmium, the characteristic structures of parameters $P_{L}, \gamma$ and $L_{\perp}$ move towards higher scattering angles with the reduction of collision energy, which agrees with FBA predictions [23].

- In contrast to the cadmium atoms in the case of the CCC model, the characteristic structures of the $\gamma$ parameter are visible for slightly larger values of the scattering angle than for the RDWA model $\left(\approx 5^{\circ}\right)$. 
- For all the examined collision energies, in the range of the scattering angle between $15^{\circ}$ and $40^{\circ}$ experimental data for the shape parameter $P_{L}$ show two minima reproduced by the CCC model. The RDWA model provides only one minimum around $35^{\circ}$. Analogous structures (two maxima for CCC and one maximum for RDWA) are visible for the angular momentum transfer parameter $L_{\perp}$.

- In the case of all impact energies, there was no significant deviation of the $P^{+}$parameter value from unity, which indicates that the examined collision process is coherent.

- It appears that in the range of examined collision energies, the CCC model reproduces experimental results better than the RDWA model.

\section{Peculiar structures in alignment angle $\gamma$}

The analysis of the obtained EICP data as a function of two parameters: the collision energy and the scattering angle, allowed us to observe the characteristic behavior of the function of the alignment angle $\gamma$. For example, in the case of zinc, it has a continuous character at $100 \mathrm{eV}$ (continuous descending) while for $80 \mathrm{eV}$ it reaches the values of $-90^{\circ}$ and $90^{\circ}$ (discontinuous ascending). Therefore, one can expect a helical structure in the range of the impact energies between $80 \mathrm{eV}$ and $100 \mathrm{eV}$ and the scattering angle close to $150^{\circ}$ (see Fig. 7). This structure results directly from the definition of $\gamma$ parameter (8) (see [24] for details).

However, it is difficult (without high-resolution theoretical data) to predict the experimental conditions for which it can be observed. Such structures, due to their very rapid character, can be used to verify theoretical models. Moreover, this

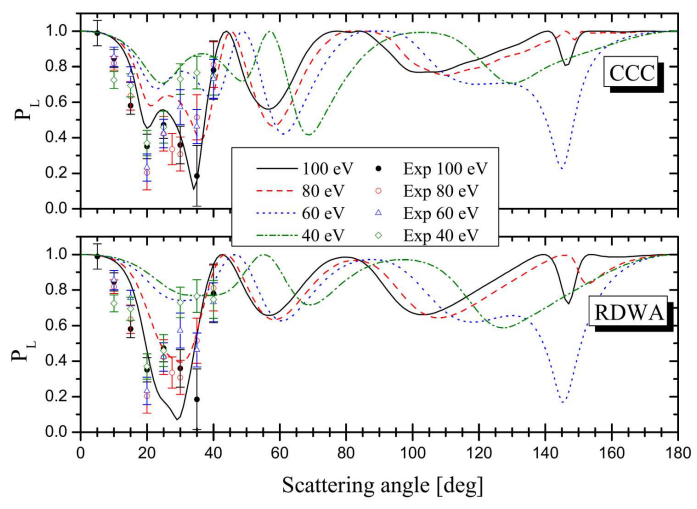

Fig. 6. Shape parameter $P_{L}$ for electronic excitation of $4^{1} P_{1} \mathrm{Zn}$ state. Experimental data: $(\bullet)$ $100 \mathrm{eV},(\circ) 80 \mathrm{eV},(\triangle) 60 \mathrm{eV},(\diamond) 40 \mathrm{eV}$. Theoretical data: (CCC and RDWA): (-) $100 \mathrm{eV},(--) 80 \mathrm{eV}$, $(\cdot \cdots) 60 \mathrm{eV},(-\cdot-) 40 \mathrm{eV}[23,26,27]$.

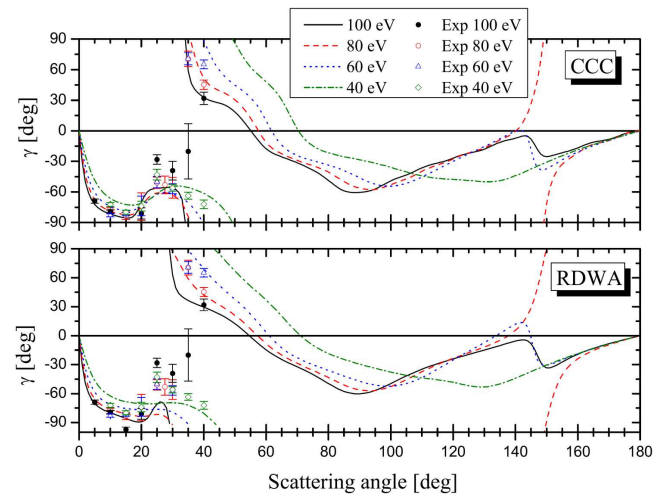

Fig. 7. Alignment angle $\gamma$ for electronic excitation of $4^{1} P_{1} \mathrm{Zn}$ state. Experimental data: (•) $100 \mathrm{eV}$, (॰) $80 \mathrm{eV},(\triangle) 60 \mathrm{eV},(\diamond) 40 \mathrm{eV}$. Theoretical data: (CCC and RDWA): (-) $100 \mathrm{eV},(--) 80 \mathrm{eV},(\cdots)$ $60 \mathrm{eV},(-\cdot-) 40 \mathrm{eV}[23,26,27]$.

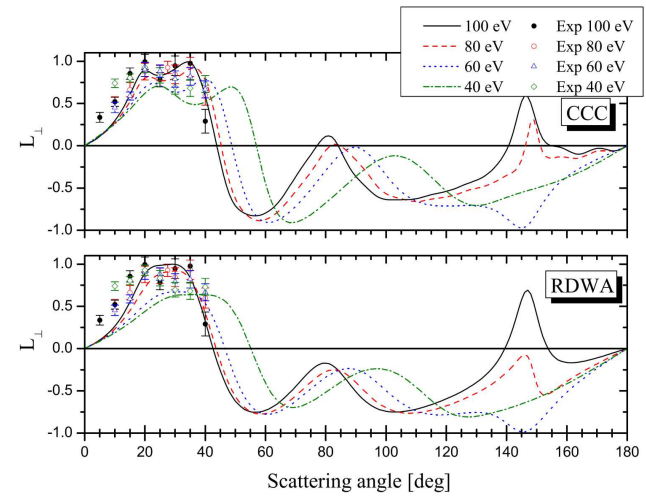

Fig. 8. Angular momentum transfer $L_{\perp}$ for electronic excitation of $4^{1} P_{1} \mathrm{Zn}$ state. Experimental data: $(\bullet) 100 \mathrm{eV},(\circ) 80 \mathrm{eV},(\triangle) 60 \mathrm{eV},(\diamond) 40 \mathrm{eV}$. Theoretical data: (CCC and RDWA): (-) $100 \mathrm{eV}$, $(--) 80 \mathrm{eV},(\cdots) 60 \mathrm{eV},(-\cdot-) 40 \mathrm{eV}[23,26,27]$.

issue is a very interesting physical situation, because for such collision conditions one of the scattering channels is completely closed. It is manifested by reaching the maximum value of the parameter determining the total angular momentum transfer $L_{\perp}$ during the collision and zeroing of the shape parameter $P_{L}$ (the cloud has axial symmetry). These structures were observed for both zinc (energy range $80-100 \mathrm{eV}$, scattering angle of $\mathrm{ca}$. equals $30^{\circ}$ and $150^{\circ}$, see Fig. 7) and cadmium atoms (energy range 60-80 eV and $80-100 \mathrm{eV}$, scattering angle of $c a$. equals $140^{\circ}$, see Fig. 4).

The conducted analysis indicated that such structures should appear also for other atoms. Unfortunately, due to the limited amount of systematic data on EICP, wider research in this area was difficult. Nevertheless, it was possible to indicate for several atoms $(\mathrm{Mg}, \mathrm{Ca}, \mathrm{Sr}, \mathrm{Ba})$ the ranges of collision energy and the scattering angles for which this structure should be observed [24]. Thus, these results may be stimulating also for other research groups. 


\section{Conclusions}

The presented results are the only sets of the experimentally determined EICP parameters for cadmium and zinc atoms describing collisional excitation to the first $P$ state. A comparison of the EICP data for zinc and cadmium allows us for the formulation of more general conclusions:

- In the zinc case, the discrepancies between theoretical and experimental results are more significant for lower collision energies, while for cadmium they remain at a similar level.

- For both elements, all the characteristic structures of parameters $P_{L}, \gamma$ and $L_{\perp}$ move towards higher scattering angles with the reduction of collision energy, which agrees with FBA predictions.

- In the case of zinc CCC predictions, the characteristic structures of $\gamma$ are observed at slightly larger scattering angles in comparison to the RDWA results, while in the cadmium case this trend is inverted.

Moreover, in the obtained data for zinc and cadmium atoms, specific structures in the alignment angle $\gamma$ were found, which are predicted by theory. These structures were also indicated in the data of magnesium, calcium, strontium and barium atoms.

\section{References}

[1] U.J. Sofia, D.M. Meyer, J.A. Cardelli, Astrophys. J. Lett. 522, L137 (1999).

[2] C.R. Silveira, B. Barbuy, A.C.S. Friaça, V. Hill, M. Zoccali, M. Rafelski, O.A. Gonzalez, D. Minniti, A. Renzini, S. Ortolani, Astron. Astrophys. 614, A149 (2018).

[3] B.P. Marinkovic, V. Pejcev, D.M. Filipovic, D. Sevic, S. Milisavljevic, B. Predojevic, Radiat. Phys. Chem. 76, 455 (2007).

[4] D. Dziczek, D. Dyl, M. Piwiński, S. Chwirot, Acta Phys. Pol. A 93, 717 (1998).

[5] D. Dziczek, M. Piwiński, M. Gradziel, S. Chwirot, Acta Phys. Pol. A 103, 3 (2003).

[6] S.A. Napier, D. Cvejanović, J.F. Williams, L. Pravica, D. Fursa, I. Bray, O. Zatsarinny, K. Bartschat, Phys. Rev. A 79, 042702 (2009).

[7] H.G. Bohachov, E.Y. Remeta, Opt. Spectrosc. 128 (2020).

[8] D. Cvejanovic, S. Napier, J.W. Williams, L. Pravica, J. Phys. Conf. Series 194, 042019 (2009).

[9] R.P. McEachran, A.D. Stauffer, M. Piwiński, L. Pravica, J.F. Williams, D. Cvejanovic, S.N. Samarin, J. Phys. B 43, 215208 (2010).
[10] B. Bederson, Comm. At. Mol. Phys. 1, 41 (1969).

[11] D. Dyl, D. Dziczek, M. Piwiński, M. Gradziel, R. Srivastava, R. Dygdała, S. Chwirot, J. Phys. B 32, 837 (1999).

[12] Ł. Kłosowski, M. Piwiński, D. Dziczek, K. Wiśniewska, S. Chwirot, Meas. Sci. Technol. 18, 3801 (2007).

[13] S. Chwirot, D. Dziczek, R. Srivastava, D. Dyl, R. Dygdała, J. Phys. B 29, 5919 (1996).

[14] M.J. Brunger, J.L. Riley, R.E. Scholten, P.J.O. Teubner, J. Phys. B 22, 1431 (1989).

[15] H.J. Beyer, H. Hamdy, E.I.M. Zohny, K.R. Mahmoud, M.A.K. El-Fayoumi, H. Kleinpoppen, J. Abdallah Jr., R.E.H. Clark, G. Csanak, Z. Phys. D 30, 91 (1994).

[16] Ł. Kłosowski, M. Piwiński, D. Dziczek, K. Pleskacz, S. Chwirot, Phys. Rev. A 80, 062709 (2009).

[17] N. Andersen, J.W. Gallagher, I.V. Hertel, Phys. Rep. 165, 1 (1988).

[18] N. Andersen, K. Bartschat, Polarization, Alignment, and Orientation in Atomic Collisions, Vol. 96 of Atomic, Molecular, Optical and Plasma Physics, Springer, Cham 2017.

[19] M. Piwiński, D. Dziczek, R. Srivastava, M. Gradziel, S. Chwirot, At. Mol. Phys. Proc. SPIE 5258, 132 (2003).

[20] M. Piwiński, D. Dziczek, Ł. Kłosowski, S. Chwirot, Eur. Phys. J. Spec. Topics 222, 2273 (2013).

[21] M. Piwiński, Ł. Kłosowski, Vacuum 156 , 154 (2018).

[22] M. Piwiński, D. Dziczek, Ł. Kłosowski, R. Srivastava, S. Chwirot, J. Phys. B 39, 1945 (2006).

[23] M. Piwiński, Ł. Kłosowski, S. Chwirot, D.V. Fursa, I. Bray, T. Das, R. Srivastava, J. Phys. B 51, 085002 (2018).

[24] M. Piwiński, Ł. Kłosowski, S. Chwirot, Acta Phys. Pol. A 134, RK.134.2.1 (2018).

[25] M. Piwiński, D. Dziczek, R. Srivastava, M. Grądziel, S. Chwirot, J. Phys. B 35, 3821 (2002).

[26] M. Piwiński, Ł. Kłosowski, D. Dziczek et al., Phys. Rev. A 86, 052706 (2012).

[27] M. Piwiński, Ł. Kłosowski, D. Dziczek, S. Chwirot, D.V. Fursa, I. Bray, Phys. Rev. A 91, 062704 (2015). 